\title{
Scientific Principles behind 2017 World Universiade of Safety Lantern and Torch
}

\author{
Wei Long Chen, Fang Lin Chao* \\ Department of Industrial Design Chaoyang University of Technology,436, Taiwan R.O.C
}

\begin{tabular}{l} 
A R T I C L E I N F O \\
\hline Article history: \\
Received: 11 May, 2018 \\
Accepted: 12 June, 2018 \\
Online: 02 July, 2018
\end{tabular}

Keywords:

Scientific principles

Safety lantern

Torch

Convection

\begin{abstract}
A B S T R A C T
The torch relay for the 2017 Taipei Summer Universiade started June. The innovative torch design was altered and tested, follow-up production successfully. For preventing weather effect, the head of the torch embedded with a unique airflow structure that allowed the sacred flame to pass the wind and rain tests. The safety lantern displays a $3 D$ spiral flash. Safety lantern uses biofuel so that the mother flame can transport by air. The scientific principles are Stack effect, Coanda effect, Bernoulli's principle, and Capillary phenomenon. Product design refers to principles and a series of work from the specifications to determine the product structure. Cross-discipline collaboration is needed to increase the integrity.
\end{abstract}

\section{Introduction}

The torch relay for the 2017 Taipei Summer Universiade started June 20 in the Italian city of Turin [1], "with a vision to shape the leaders of tomorrow through their experiences of international student sport." It was then passed by Professor Gianmaria Ajani, the rector of the University of Turin, to Matytsin. He then transferred the flame to the Taipei 2017 Torch, which was held by Ko Wen-je, the Mayor of Taipei (Figure 1). The domestic leg of the Torch Relay took the flame and visited 22 cities and counties.

The unique design developed by cooperation of both designers and engineers. The torch design included Taiwan's essential design elements and craft skills. With the collaboration of scientist and designer, the concept was revised and tested, follow-up production successfully [2]. For preventing weather's affection, the head of the torch embedded with a unique airflow structure that allowed the sacred flame to pass the wind and rain tests. The safety lantern displays a single three-dimensional spiral flash (Figure 2).

Safety lantern have several vertical vanes, each vane has a section encircled by the concealing member defining a flow accelerating zone. External air flows into the flow diverting mechanism through the air intake ports, circumferentially conceals with the wall.

Figure 2 also shows the concept of torch design comes from Confucian's article "Gentlemanly fair play." The Master said,

${ }^{*}$ Corresponding Author: Fang Lin CHAO, Email: flin@cyut.edu.tw
"Supportive, encouraging, congenial - such a man may be called a gentleman." Fair play induces supportive and encouraging with a player, congenial with friends.

The torch is made of metal, on which there are an array of ventilating holes. The lower holding portion made of weaving bamboo comes from eastern civilization.

The safety lantern keeps the mother flame continuously so that the fire can pass through the different location. The design includes a safety feature to avoid accidental burning if the fuel leaks out. There is no CO generated during combustion, so the safety lantern can transport using commercial air traffic.

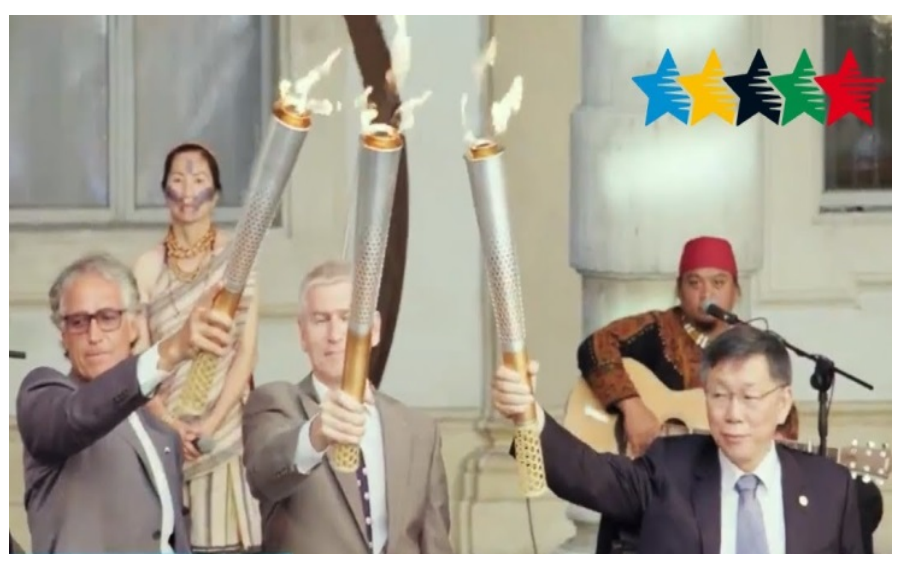

Figure 1. Torch used in 2017 Taipei Summer Universiade [2] 


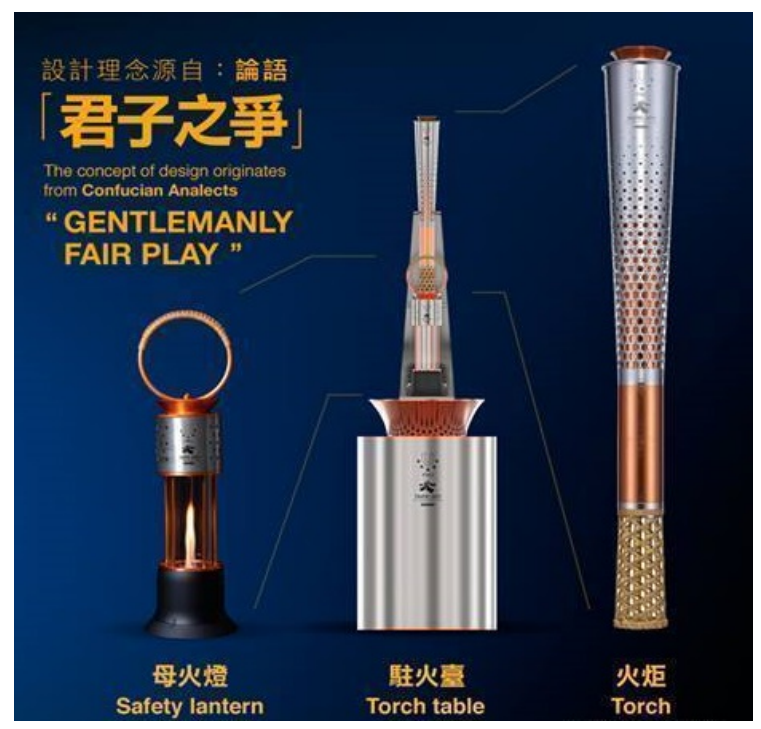

Figure 2. The concept of torch design comes from Confucian [2], design team: H.L Chang of Ui-D Taiwan. The safety lantern keeps the mother flame continuously, the torch table presenting the flash at the main venue of the sports competition, and the relay torch. The height of safety lantern and torch are $50 \mathrm{~cm}$ and $70 \mathrm{~cm}$.

\section{Scientific Principles}

The spiral frame of safety lantern is distinct and has a stable shape and an elongated height (Figure 2). Air driven by buoyancy goes upward and draws in fresh air through the passages which make air flowing therein rotate and go upward.

For the torch, after gas and air mixing process and is being ignited by the igniting needle, combustion flame flowing out from the top cap and fresh air drawn from the side holes for mixing. Gas and air mixture undergoes the mixing processes and flows out of the plurality of exit.

\subsection{Stack effect}

The safety lantern has a concentrated hot zone around and above the combustion head due to combustion flame and spiral flow around. Heated air with lower density in the heated region flows upward and creates low pressure to draw fresh air from intake port into the transparent shield, thereby increasing the height of flame in the torch. This phenomenon is known as stack effect or chimney effect.

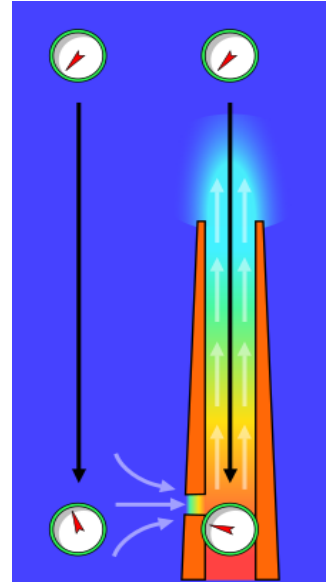

Figure 3. The safety lantern: stack effect [3] (gauges represent absolute air pressure, airflow indicates with grey arrows)

\subsection{Coanda effect and centrifugal force effect}

The passages direct fresh air toward the inner surface of a transparent shield at a specific angle to create a spiral air flow pattern and tangent to the inner surface of the shield. This feature dramatically helps to stabilize swirling air flow pattern due to Coanda effect and centrifugal force.

Coanda effect [4] related to the tendency of a fluid jet to stay attached to a convex surface. The pressure difference across the jet causes the jet to deviate towards the nearby surface Figure 4, and then to adhere to it. The separation angle is a function of the Reynolds number of the fluid.

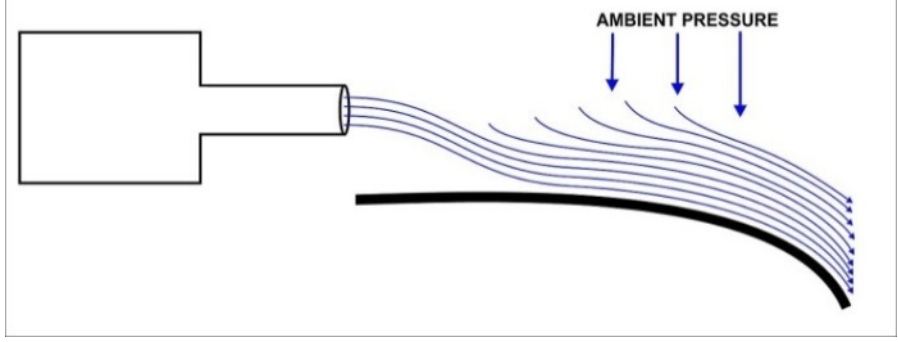

Figure 4 . The jet will cling to the surface because any molecules of ambient air that move into the space between the jet and swept away, adhere to the surface even if it is curved [4].

The fluid stream attaches a contour when the flow is directed at a tangent to that surface. This spiral motion of air flow also induces centrifugal force. The safety lantern takes advantages of stack effect and Coanda effect which help to create a stable spiral and elongated flame. The centrifugal force is an inertial force directed away from the axis of rotation that appears to act on objects when viewed in a rotating reference axis [5]. In a rotating reference frame, objects appear to be under the influence of a radially outward force that is proportional to their mass, to the distance from the axis, and to the square of the angular velocity [4]. The detail of structure in the safety lantern shows in Figure 5.

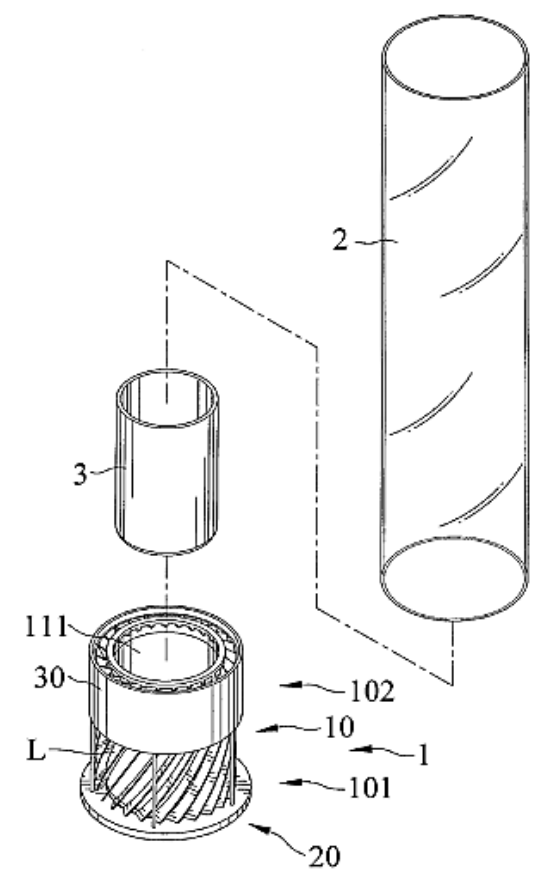

Figure 5 . The safety lantern [7]: a perspective view of the flame device, where 1: assembly, 2: glass tube, 3 : container, 20: basement, 30: cover guider, 10: passages, 101: inlet end, and 102: acceleration zone. 


\section{W.L. Chen et al. / Advances in Science, Technology and Engineering Systems Journal Vol. 3, No. 4, 21-25 (2018)}

The safety lantern produces a swirling flame, and a stable fluid creates within the guided shield channels. Heated air in the shield creates buoyancy due to a difference in air density. The perspective view (Figure 5) showing the flame device producing a stable and prolonged flame in a steady fluid field. Undergoes convection directs air through the transparent glass shield (\#2). Centrifugal forces also keep air moving spirally and drive air into the safety lantern.

\subsection{Capillary phenomenon}

The capillary phenomenon is the ability of a liquid to flow in narrow spaces in opposition to gravity [6]. The effect can find in the drawing up of water between the hairs of a paint-brush. If the diameter of the tube is sufficiently small, then the combination of surface tension and adhesive forces dominate. The thinner the space in which the liquid can travel, the further up it goes. In torch design, we choose liquid transparency "biofuel" as fuel.

The capillary phenomenon presents while the biofuel's viscosity is low. This property allows the fuel transport from the fuel tank to the upper ignition area. When fuel is filled into the body below the wick, the wick can volatilize fuel for combustion. The cord placed in the holding space and the two jaws can hold the wick so that the wick's head end located in the burn area [7]. The user can turn to adjust the elastic, change the clamping gap when clamping the cord will be compressed. Adjust the speed at which the wick draws fuel, thus controlling the size of the combustion flame. The Use of metal mash wick also removes the disadvantage of waste and burn out of traditional cotton thread core.

\subsection{Bernoulli's principle}

Bernoulli's principle states that an increase in the speed of fluid co-occurs with a decrease in pressure. Bernoulli's equation is sometimes valid for the flow of gases: provided that there is no transfer of kinetic or potential energy from the gas flow to the compression or expansion of the gas. Bernoulli equation applies to incompressible fluids, and steady compressible fluids up to approximately Mach number 0.3 [8]. There are many applications in surrounding life, such as an injector on a steam locomotive. The pitot tube is used to determine the airspeed. The flow speed of fluid can also be measured using the Venturi meter [9]. The pressure in the reduced diameter region decreases and display in the meter by liquid height difference.

The main body of the torch is an aluminum metal rod. The hollow metal tube contains the gas supply lines. The high-pressure gas is conveyed upward by a metal pipe from a fuel tank in below. The upper section has an enlarged gas mixing zone. At the top of the gas mixing zone is the metal flame exit cover. The highpressure gas gradually spreads to the top metal cover of the torch. As shown in Figure 6, the central hole on the metal cover is the exit of the main flame. There are four big holes next to it, which is the side flame exit. When the gas in the torch ejectes at high speed, the pressure in the central area becomes smaller; therefore, the external air will flow from the side of the leaf-shaped opening into the mixed gas area (Figure 7). When the outside air enters the mixed gas zone, it can also cool the aluminum rod; it avoids the chance of being exposed to the outside of the torch.

The surrounding metal shell wrapped on the outside of the aluminum metal tube. The hand grip is under the metal shell, and the grip is a structure woven with bamboo. Burning the flame increases the temperature of the aluminum metal tube. There is an air layer between them (Figure 7), the thermal conductivity of the air is very low which reduced the amount of heat transfer between internal pipe and the periphery. When cold air enters from the intake holes, it also cools the metal pipes at the same time.

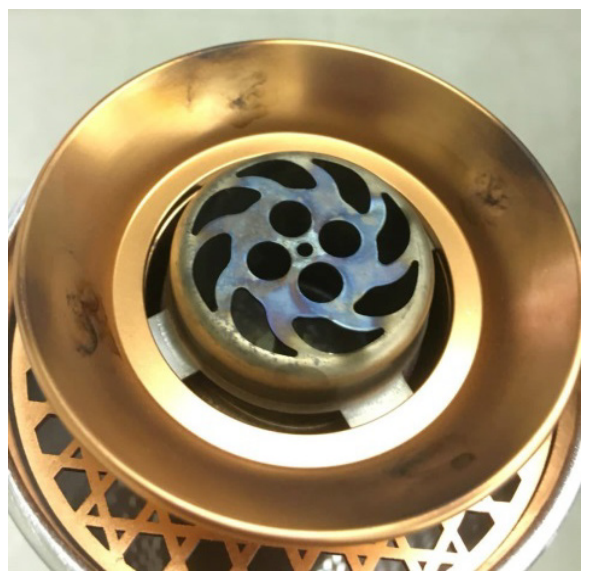

Figure 6. The metal cover on top of the torch: The central hole is the exit of the main flame. There is four side flame exit. The outer leaf-shaped opening is air inlet of the mixing zone.

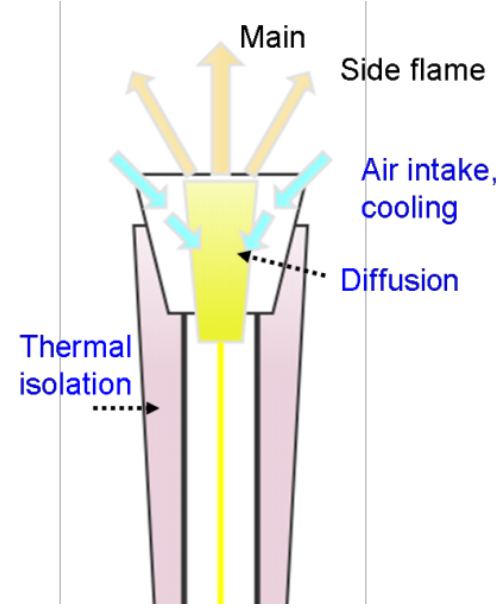

Figure 7. Torch: External airflow driven by Bernoulli's principle, where the gas ejects and spreads (diffusion). The low pressure in the central area causes the external air enters the air intake holes into the mixing zone. The air between the aluminum rod and surrounding metal decoration also provide thermal isolation.

\section{Experimental Evaluation}

In the previous chapter, we discussed the scientific principles behind the design of the torch. In this chapter, we utilize two prototypes and perform functional tests. The purpose of the experiment is verifying the benefits of the above scientific principles in design. The prototype of the product is one-to-one scale and tested according to the conditions of use. In addition to the shape of the flame, we also care about the safety of the user, and we hope to appropriately lower the temperature of the product in areas that may be in contact with user's body.

\subsection{Safety Lantern}

Figure 8 indicates the evaluation of the experimental prototype. We observed safety lantern with spiral shape and an elongated height. As the air is heated, it rises along the guide channel, and the airflow is a steady laminar flow. Because of the Coanda effect, the airflow is attached to the inner wall of the glass and rotates upwards. As the stack effect and centrifugal force continue to rise 
upward, a stable flash formed in the lower half zone. After passing through the middle area, transition and turbulent zone take place, and the shape of the flame is irregular.

The combination of the mentioned effects can substantially elongate the height of flame and change the swirling speed of flash according to flame visualization required. If the flame guiding member is absent, it is difficult to control a smooth flow. The flame guiding member allowed controlling natural convection and drawn fresh air from outside the chamber. The flame guiding member of 44-degree skew angle shows a reasonable display quality (Figure 8 ). When cold air is introduced, it also cools the metal and glass tube. The temperature measurement of aluminum castings and iron plate indicated the contact temperature is not higher than 60 degrees $\mathrm{C}$.

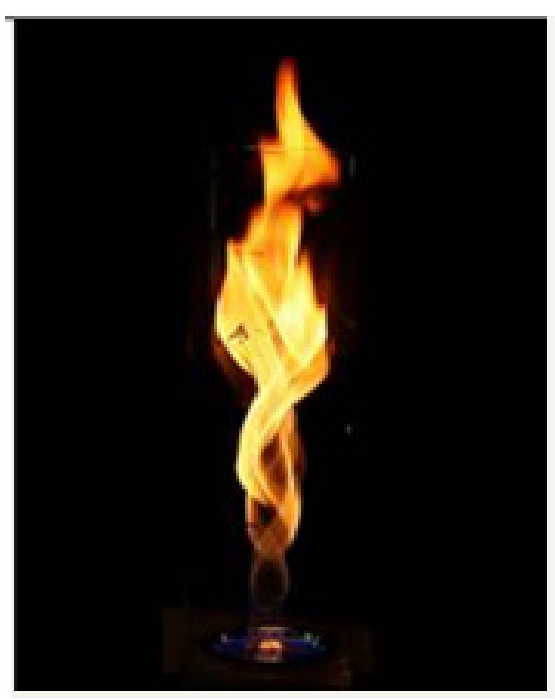

Figure 8. Evaluation of experimental prototype. There are three flame zones; laminar zone (lower), transition zone (middle), and turbulent zone (upper).

\subsection{Torch}

There are five flame outlets above the fire exit, which scatter at different angles respectively to show the flame shown in Figure 9 and 1 . The runner at the fuel outlet must be balanced so that the flame maintains a similar height in all directions. It successfully demonstrated the five lights which symbolizing the Olympic Games.

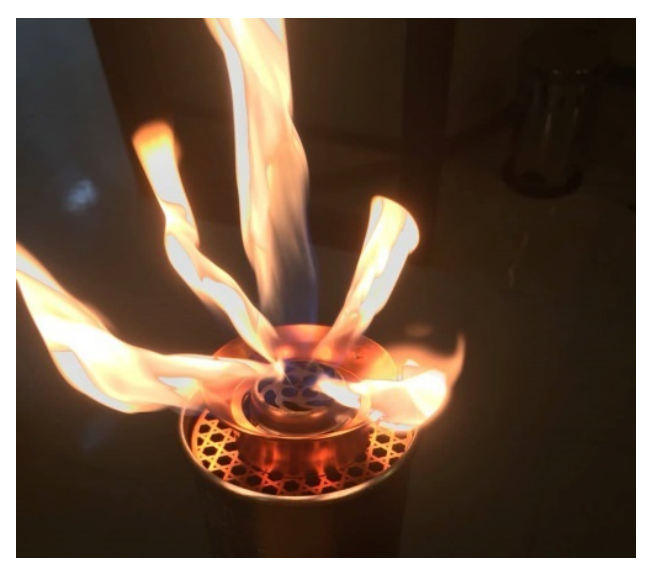

Figure 9. The flow diverting mechanism design of the torch

At the previous trial, the torch flame that burns on mixing gas from first mixing process undergoes convection directly with air, thereby creating a more significant portion of colorless and transparent flame, causing a less visible swirling pattern and shorter in flame height. We will expand the gas mixing area at the gas outlet, let the gas mix and send it out from the top hole. After gas and air mixed in the chamber, the torch shows a reasonable display quality.

\section{Cross-discipline Collaboration}

Although scientific principles are well known, designer seldom can integrate. Product design refers to a series of work from the specifications to determine the product structure. The designer must take into account the form, technology, and regulations, through the appropriate product form to reveal differentiated value.

\subsection{Integration}

We experienced cross-discipline collaboration is vital to torch design. It covers material, combustion, fluid dynamics, heat transfer, usability, and aesthetics. One of successful field practice project is EU-IDES-EDU: "Master and Post-Graduate education and training in multi-disciplinary teams." In [10], describes the necessity of more integrated and cross-disciplinary approaches to building design. The challenges encountered during implementation of the design education across different professions were discussed. The Integrated Design Process (IDP) was developed to streamline the design process of solar integrated low-energy buildings. The involvement of engineers in early stage improved design significantly.

We also experienced idea jumping iteratively through the stimulus of CAE simulation and prototype experiment during torch design. In [11] authors present "speculative design as a means to technological fluency." Speculate means form a theory or conjecture about a subject without firm evidence. Creativity's natural imply explore and discover a new connection between facts or knowledge. Firm evidence is only a stepping stone to problemsolving. Technological fluency is a term used to describe a level of expertise: the capability to understand, use, and assess technology beyond its rote application. By using a Speculative Design approach to extend the idea of technological fluency in new directions, broadening understandings of the development of technology and adding a dimension of criticality [12].

Design often plays a central role in technology fluency programs. The design process typically derived from engineering approach in which functional requirements drive the process toward the solution. Speculative design project involves the construction of prototypes. The education of the designer's technological imagination consists in learning how to use tools, expression, techniques for rapid prototyping, processes of iterative experimentation, and skills in social negotiation and integration [12].

Cross-disciplinary and integrative are significant attributes of technological fluency in speculative design projects. Pressures internal and external to engineering courses for the adoption of multidisciplinary team-based work are discussed. Some key factors arising from the survey are addressed [13].

\subsection{The design process and CAE}

The critical elements of renovation in torch design are related to cross-discipline and knowledge. As shown in Figure 10, the design guide help prevent mistake arrangements. CAD/ CAE are used to realize the idea quickly. 
There are many engineering issues in torch design; such as heat transfer, fluid dynamic, mechanical stress and other physical principles. The flow field simulation enables fine tune of geometric design. Functional test proof and confirm the product features, through a series of measurements such as thermal energy, flame height, contact temperature (glass and metal). After that, the checklist and regulations were reviewed to enable the modified concepts.

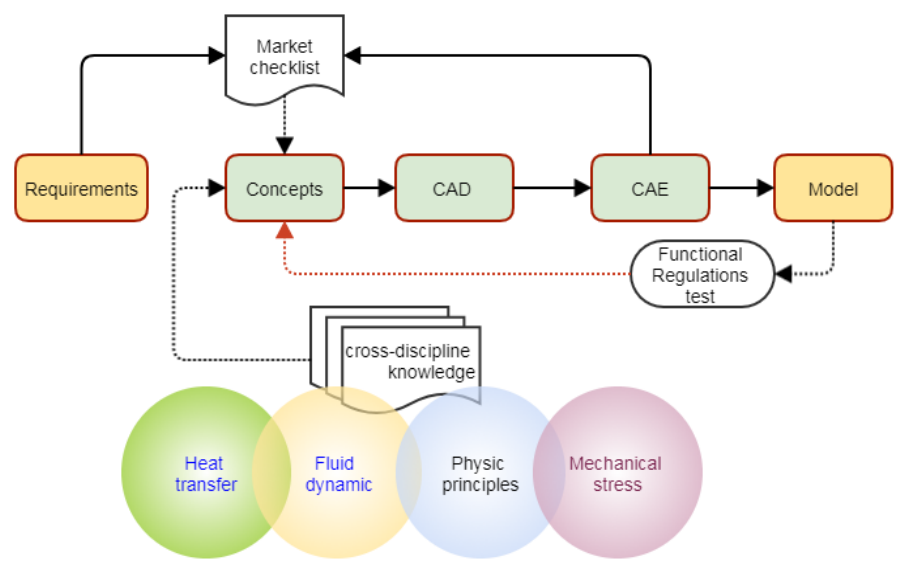

Figure 10. Design process

We built the digital model with CATIA. As shown in Figure 10 , the prototype model can utilize for measuring actual physical parameters. Comparing the measured data and simulation results, we refine the CAE models. When the settings are confirmed, the designer can use the digital model to obtain design parameters. After that, a functional test proof and validate the product features. The cross-discipline knowledge help prevent contradiction design. The CAE is used to verify the idea quickly and shortens development cycle time.

The role of a designer is changing according to the evolutional technology and society. From the torch project, product renovation needs to cooperate with others expertise closely. Understanding the relevant principles in the product can lead to support through cross-domain collaboration.

\section{Conclusions}

In this article, we discussed the scientific principles within the torch and safety lantern with spiral shape and an elongated height; namely Stack effect, Coanda effect, Bernoulli's principle, and Capillary phenomenon. After gas and air mixing process and is being ignited, combustion flame flowing out from the grooves and fresh air drawn from the passages mix. Air in the shield driven by buoyancy goes upward and brings in fresh air through the passages which make air flowing therein rotate and go uphill.

The process of design also provided. We employee qualitative and CAE design process to fulfills its primary and auxiliary functions. Design process helps understand combustion phenomenon and trend. Through the model test, designer and engineers can select proper parameters. Cross-discipline collaboration is needed to increase the integrity of the concept. Designers should experience the scientific principles to collaborate with other team members in cross-domain development.

\section{Acknowledgments}

We wish to thank design effort of Ui-D, and students in CAE Lab in Chaoyang University of Technology for supplying the testing and measurement. This research partially supported by the ProIroda company with prototyping.

\section{References}

[1] https://www.insidethegames.biz/articles/1051799/taipei-2017-torch-relaybegins-in-turin

[2] https://udn.com/news/story/11406/2671767

[3] https://en.wikipedia.org/wiki/Stack_effect

[4] https://en.wikipedia.org/wiki/Coand\%C4\%83_effect

[5] https://en.wikipedia.org/wiki/Centrifugal_force

[6] https://en.wikipedia.org/wiki/Capillary_action\#Phenomena_and_physics_o f_capillary_action

[7] Chen, Wei-Long, and Jan-Nan Chen. "Device for producing stable and augmented flame." U.S. Patent 8,641,413, issued February 4, 2014. https://patents.google.com/patent/US8641413B2/en

[8] White, Frank M. Fluid Mechanics, 6th ed. McGraw-Hill International Edition. p. 602, 2017.

[9] Kamela, M., "Thinking about Bernoulli," The Physics Teacher, 45(6), 379381, 2007. https://doi.org/10.1119/1.2768700

[10] C., Brunsgaard, P. Dvořáková, A. Wyckmans, M. Laskari, "Integrated energy design-Education and training in cross-disciplinary teams implementing energy performance of buildings directive (EPBD)", Building and Environment, 72, 1-14, 2014. https://doi.org/10.1016/j.buildenv.2013.10.011

[11] Lukens, Jonathan, and Carl DiSalvo. "Speculative design and technological fluency." International Journal of Learning and Media, 3(4), 23-40, 2011. doi:10.1162/IJLM_a_00080

[12] Burdick, Anne, and Holly Willis. "Digital learning, digital scholarship and design thinking." Design Studies, 32(6), 546-556, 2011. https://doi.org/10.1016/j.destud.2011.07.005

[13] Denton, H. G. (1997). Multidisciplinary team-based project work: planning factors. Design Studies, 18(2), 155-170, 1997 . https://doi.org /10.1016/S0142-694X(97)85458-0 